\title{
Auditory Guidance System for the Blind with Signal Transformation from Multiple Ultrasonic to Binaural Audio
}

\author{
Young-Jip Kim*, Chong-Hui Kim**, and Byung-Kook Kim**
}

\begin{abstract}
We design an auditory guidance system for the blind using ultrasonic to audio signal transformation. We first investigated system requirements, and designed a simple but useful portable guidance system for the blind. The system derives visual information using multiple ultrasonic sensors, and transforms it to binaural auditory information using a suitable technique. The user can recognize the position of obstacles and the surrounding environment. The system is composed of two parts. One is glasses type system and the other is cane type with guide wheels. The first functions as an environment sensor, and the latter functions as a clear path indicator. Wide beam angle ultrasonic sensors are used to detect objects in broader range. The system is designed for a battery supplied portable model. Our design is focused on low power consumption, small size, lightweight, and easy manipulation.
\end{abstract}

Keywords - Signal Transformation, auditory guidance system, broad beam ultrasonic sensor, clear path indicator.

\section{INTRODUCTION}

A $\mathrm{S}$ life becomes more abundant, the desire of human welfare is being increased. Hence interests in the life quality becomes high not only for normal people but also for handicapped people. Handicapped people that have problems in the sensory systems live through various hardships, since human protect themselves from danger and respond to external stimuli with recognition of environmental information. Visual data contains a large amount of information such as shape, color, roughness, and motion of objects. That is, the visual sensory system receives much more environmental information than any other sensory system. Hence, the visual system is very important for not only handicapped persons but also non-handicapped persons. In this point of view, a visually impaired person can obtains even much less information than any other kind of disabled person. These drawbacks restrict their social and economic activities. Hence, the visual substitution system can augment their scope of social and economic activity. Therefore, the welfare of handicapped person may be moved up.

Rehabilitation professionals often divide individuals with such visual deficits into two categories [1]: the functionally blind (those with no vision or only light/dark perception) and those with residual "low vision" (reduced visual acuity and/or abnormal visual fields uncorrectable by conven-

Young-Jip Kim, Chong-Hui Kim and Byung-Kook Kim are with the Real-Time Control Laboratory, Department of Electrical Engineering \& Computer Science, Korea Advanced Institute of Science and Technology (KAIST), 373-1 Kusong-dong, Yusong-gu, Taejon 305-701 Korea

Email: yjkim@rtcl.kaist.ac.kr*, chkim@rtcl.kaist.ac.kr**, bkkim@ee.kaist.ac.kr*** tional means). In the former case, the search is for an appropriate substitute; while in the latter, one attempts to make the best use of whatever visual function remains by providing an appropriate low-vision aid. Such a device can be optical (using the optical properties of lens, prism, and/or mirror combinations to enhance performance), nonoptical, or electro-optical (electronic).

In this paper, we present an Electronic Travel Aid (ETA) that can provide visual information needed for walking of visually impaired persons. Ultrasonic sensors are used to acquire environmental data. The analysis of the acquired data provides visual information, and the user can get the information through binaural audio output.

The rest of paper is constructed as follows. Section II gives the previously development devices and their features. Section III describes the system requirement of a portable guidance system for the blind. Section IV describes system design concept, system elements, and signal transformation algorithm. Section V contains results of basic preliminary experiments. Finally, Section VI concludes this paper.

\section{PREVIOUS WORKS}

A serious problem for visually impaired persons is hardship of walking. The most widely used method is a white stick. A person should train for a long time to use a white stick. The other solution is a guide dog, which is very expensive, but can not provide so much information.

Hence during the last several decades, several models of blind mobility aids have been developed. Sonic-torch [2], Sonicguide [3], Sonic PathFinder [4], Mowat Sensor [5], Nottingham Obstacle Detector [6], Laser-Cane [7], and GuideCane [8] are called clear path indicators or obstacle detectors since the blind can only know whether there is an obstacle in the path ahead [9]. These devices are used to search for obstacles in front of the blind person, and they operate in a manner similar to a flashlight, which has very narrow directivity. Sonic-Guide and Navbelt [10], however, are called an environment sensor since it has wide directivity enabling it to search for several obstacles at the same time [11].

SonicGuide [12] is an obstacle detector: It consists of a pair of spectacles with an ultrasonic echolocation system allowing evaluation of azimuth and distance of obstacles. SonicGuide comes in the form of a pair of spectacle frames, with one ultrasonic wide beam transmitter mounted between the spectacle lenses and two receivers on both sides of the transmitter. Signals from the receivers are frequency 
shifted and presented separately to left and right ears. The resulting interaural amplitude difference allows the user to determine the direction of the incident echo and thus of an obstacle. The distance to an object is encoded in the frequency of demounted low-frequency tone.

The Laser-Cane [7] is a traditional long cane coupled with an infrared echolocation system aimed at detecting targets complementary to the cane. It is based on optical triangulation with three laser diodes as transmitters and three photo-diodes as receivers. The Laser-Cane can detect obstacles at head-height, drop-offs in front of the user, and obstacles up to a range of $1.5 \mathrm{~m}$ or $3.5 \mathrm{~m}$ ahead of the user.

The Mowat Sensor [5] is a hand-held ultrasonic-based device that informs the distance to detected objects by means of tactile vibrations. The frequency of the vibration is inversely proportional to the distance between sensor and object. The Nottingham Obstacle Detector (NOD) [6] is a hand-held sonar device that provides an auditory feedback, in which eight discrete levels of distance are distinguished by different musical tones.

The Navbelt [10] is a portable device equipped with ultrasonic sensors and a computer. The Navbelt provided two modes of operation. In the image mode, the Navbelt produced a 120-degree wide view of the obstacles ahead of the user. This image was then translated into a series of directional (stereophonic) audio cues through which directions were blocked by obstacles and which directions were free for travel. Another mode of operation was called guidance mode. In this mode, it was assumed that the system knew the traveler's momentary position and the traveler's desired target location. Under these conditions, the Navbelt only needed to generate a single signal that indicated the recommended direction of travel.

However, it is difficult to walk using only these devices, since they can not provide entire information. Hence they have been used only assistant methods of a white cane or a guide dog.

One of exciting possibilities for the enhancement of residual vision is image processing. Current publications in the field take one of two approaches: compensation filtering to increase the amount of useful information in the visual scene [13], or spatial remapping to shift important information to better functioning portions of the visual field [14]. The former requires hardware implementation of convolution kernels, which compensate for an individual's reduced sensitivity to various spatial frequencies. The latter requires hardware implementation of pixel shifting algorithms, which are mapped according to the patient's visual field defects. Currently, these systems are very bulky and expensive; moreover, experimental results have been interesting, but not revolutionary.

\section{REQUIREMENTS}

Some design problems common to all assistive devices include the overriding need for practicality and user acceptance. However, these factors can easily become submerged or even forgotten in the engineer's enthusiasm for the technological solution [15]. After great effort has been exerted in devising a technologically sophisticated and elegant solution, it is sometimes discovered that the intended user finds the resulting device too much trouble to use, too expensive, or too complex.

Portability, reasonable cost, and above all simplicity of controls are most important factors which govern the practicality and user acceptance of such devices. Most present models are not practical because theses systems are developed from functional and technical point of view. Hence some systems are too much expensive, complex or not proper for the portable system in the point of weight and size.

The ETA is a kind of portable device. Hence it should be a small-sized and lightweight device to be proper for portability. The visually impaired are not able to see the display panel, control buttons or labels. Hence the device should be easy to control: No complex control buttons, switches and display panel should be present. Moreover, the ETA device should be low-price to be used by more visually impaired persons.

Our Electronic Travel Aid (ETA) is designed for portable (small size and lightweight), inexpensive and easy to use, and low-power consumption (supplied by battery).

$$
\text { IV. DESIGN }
$$

\section{A. Overview}

This system is an auditory guidance system for the blind using ultrasonic to audio signal transformation. The system gathers environment data using ultrasonic sensors and extracts the visual information from the data. This visual information is transformed into audio signal to be notified. The user can recognize the environmental information through binaural sound generated by the system. The concept of our system is shown in Fig. 1.

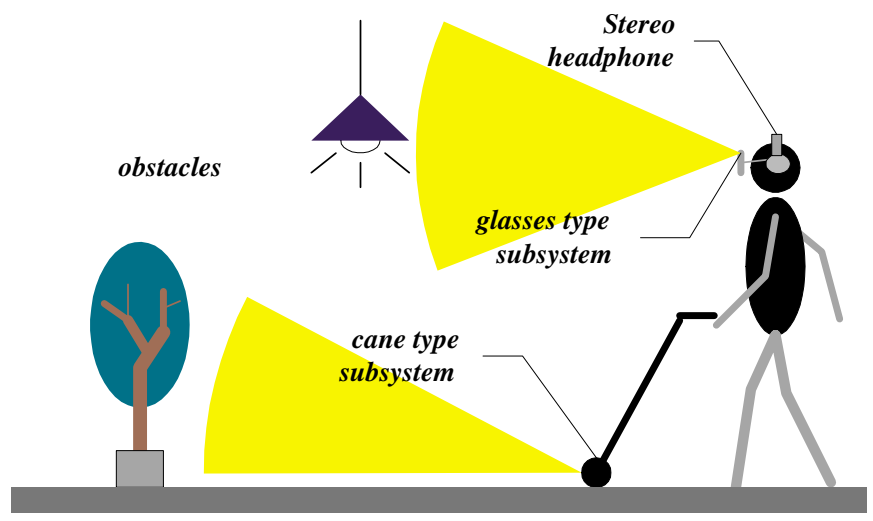

Fig. 1. The concept of the Electronic Travel Aids

To implement the previously mentioned concept, the system needs the following functions to work as an ETA. First, the system functions as a clear path indicator. This function is a basic feature that informs the user whether there is an obstacle in the path of movement.

The second function is environment recognition. A pedestrian needs to know what is beside of him to pro- 
tect himself from danger. Also the function of environment recognition can provide a help to guess where he is.

The last, the function of transformation from visual information to audio signal is needed. Auditory sensory system can provide the fastest method for a visually impaired to get external information. In our system, we use not language but sound to express the distance and direction of obstacle so that the blind can recognize the position of obstacle by intuition as fast as possible.

In our system, two types of equipment are devised for visual data acquisition. One is glasses type subsystem which put on user's head. Two ultrasonic sensors are equipped on the rims of glasses. We can extract the position data of obstacle using stereology. Since the user can move freely his head, he can confirm the path clearness and recognize obstacles in various directions.

The other is cane type subsystem which is equipped with ultrasonic sensors and wheels. The user walk with holding this cane type system in front of him like the white cane. The cane type system notifies whether any obstacle is in the middle of the walking direction. Since the wheels are always contacted with ground, the user can recognize the condition of ground such as depression, cavity, and the stairs with his hand's tactile sensation intuitively. Also the disapproval against a new equipment can be overcome by maintaining a conventionally used device of stick.

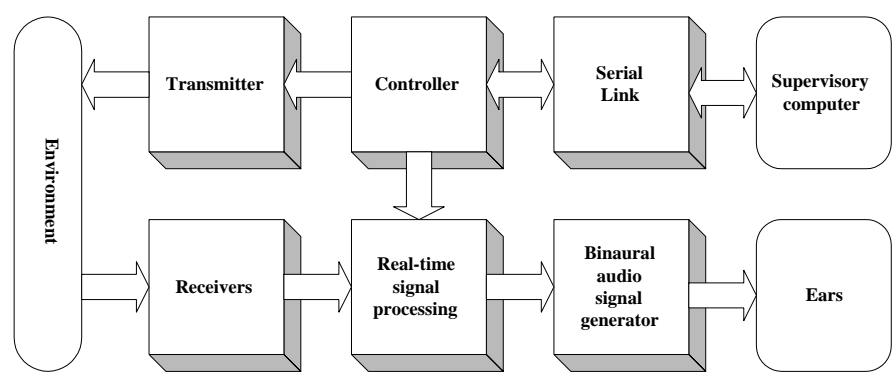

Fig. 2. Auditory guidance system using multiple ultrasonic sensors to audio signal transformation

The system is consisted of a microcomputer board, ultrasonic sensor interface, and binaural sound generator as Fig. 2 shows. These three parts compose a portable ETA system, and can be connected to the optional suprevisory computer through serial link.

\section{B. System Architecture}

Our system is built around a 16-bit $A M 188^{T M} E S$, high performance, 80C188-compatible, 16-bit embedded microcontroller with 8-bit external data bus and 32 programmable parallel I/O pins. The CPU board is small size of $56 \mathrm{~mm}$ by $65 \mathrm{~mm}$ and consists of $40 \mathrm{MHz}$ clock, 1-Megabit ROM, 1-Megabit SRAM, three internal and external programmable interval timers and three switchs. One switch is used to reset the processor, another is used to execute program in ROM and the other is used to execute program in RAM. The address and data bus can be connected to external devices with onboard sockets. This architecture makes the CPU board more flexibly applicable. The CPU board operates at $40 \mathrm{MHz}$ within $5.0 \mathrm{~V}$ plus or minus $5 \%$. Typical power consumption is $1.239 \mathrm{~W}$ at $5.25 \mathrm{~V}$ and 40 $\mathrm{MHz}$.

ROM is divided into four regions, boot, monitor, user program, and user data. There are two reset states: cold reset and warm reset. The former is to initialize processor by supplying power and the latter is to initialize processor by sending the reset signal to the CPU. The program in RAM is not erased by warm reset operation. If cold or warm reset occurrs, CPU reads and executes instructions from boot strap. By the instructions in boot strap, CPU jumps to boot program's start address and executes boot program. The boot program initializes segments and registers, copies monitor program from ROM to RAM and jumps to monitor program's start address. Then monitor program is executed. In our system, monitor and user program are executed in RAM after copying from ROM to RAM, since ROM requires longer access-time than RAM.

The software is programmed using the Borland C compiler on PC. The object machine code from the compiler can be directly used in the $A M 188^{T M} E S$ without any conversion. Using monitor program, the system's status can be monitored by PC through serial port. We can dump memory and register values, and modify the value of specific memory address. Program can be downloaded to RAM through serial port. Also we can burn the onboard ROM without ROM-writer. This comfortable environment makes it easy to debug program errors. Hence, the total system developing time can be reduced.

\section{Visual Information Acqusition}

Four wide-beam ultrasonic transceivers are used to recognize the environment. The ultrasonic sensors are designed by Murata Manufacturing Co. Ltd. and can be used both as transmitter and receiver. The ultrasonic transceiver has the following features.

i) Wide-beam angle: 40 degrees

ii) Small size: $16 \mathrm{~mm}$ diameter

iii) Light-weight: only $2 \mathrm{~g}$

iv) $40 \mathrm{KHz}$ resonant frequency

v) High reliability

The directional response of the ultrasonic sensor is shown in Fig. 3.

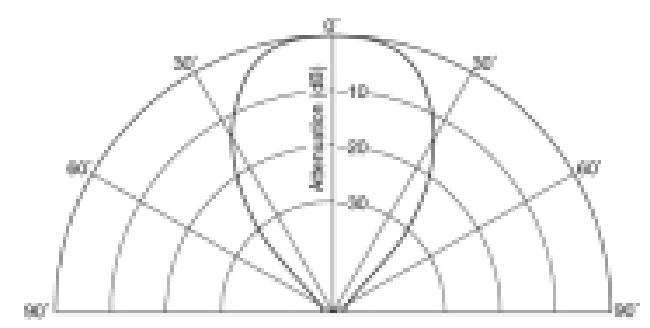

Fig. 3. Directional response of ultrasonic sensor

In our system, ultrasonic transceivers are arranged as follows. 
- Two ultrasonic transceivers of glasses type will be attached to the rims of glasses as wide as the width of glasses (approximately $15 \mathrm{Cm}$ ).

- Two ultrasonic transceivers of cane type will be attached to the cylinder like body (approximately $15 \mathrm{Cm}$ ).

As shown in Fig. 4, signal transmission and reception of the four ultrasonic transceivers are coordinated by the microcontroller through each separate transmitters and receivers. Since rechargeable batteries, approximately $5 \mathrm{~V}$, operate ETA, all elements are operated in single power supply except transmitter.

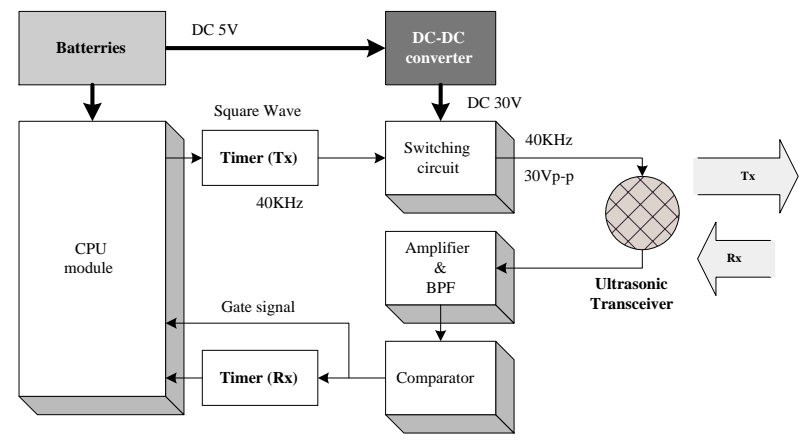

Fig. 4. Block diagram of the ultrasonic sensor interface

Our ultrasonic transceiver allows maximum 100Vp-p input voltage without bias for more wide detection range, but power consumption is very important in battery operated portable system. Hence we compromise between each other and apply $\pm 15 \mathrm{~V}(30 \mathrm{Vp}-\mathrm{p})$ input voltage to transmitter and transceiver fires 15 pulse train. After firing, if echo signals are received by transceiver, the signal is amplified and filtered, and compared with a threshold voltage above operational amplifier's bias. The comparator output signal generates hardware interrupt and is applied to timer $(\mathrm{Rx})$. Since ultrasonic transceiver generates false echoes by firing pulse train and ultrasonic transceiver's ringing time (about $2 \mathrm{~ms}$ in our ultrasonic transceiver), during $3 \mathrm{~ms}$ interruptsuppress signal is applied to receiver to ignore interrupt by false echoes. When interrupt is occurred, CPU reads timer value and computes the time of flight (TOF).

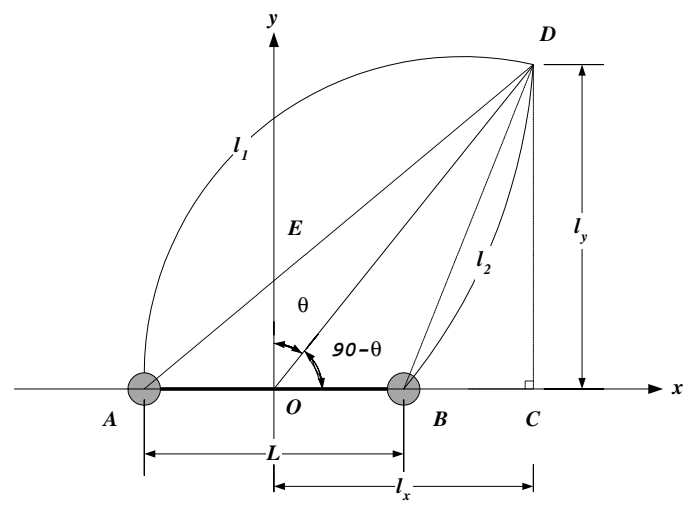

Fig. 5. Directional measurement of stereo ultrasonic sensors

Using TOF, distance and direction angle from the obstacle is computed by following equations:

$$
\begin{aligned}
& \left(l_{x}+\frac{L}{2}\right)^{2}+l_{y}^{2}=l_{1}^{2} \\
& \left(l_{x}-\frac{L}{2}\right)^{2}+l_{y}^{2}=l_{2}^{2} \\
l_{x}= & \frac{l_{1}{ }^{2}-l_{2}{ }^{2}}{2 L} \\
l_{y}= & \sqrt{\frac{l_{1}^{2}+l_{2}^{2}}{2}-\frac{L^{2}}{4}-l_{x}{ }^{2}} \\
\theta= & 90^{\circ}-\operatorname{atan} 2\left(l_{y}, l_{x}\right)
\end{aligned}
$$

where $L$ is the distance between each transceiver, $l_{x}$ is distance from center to obstacle along $\mathrm{x}$-direction, $l_{y}$ is distance from center to obstacle along y-direction, and $\theta$ is direction angle from center to obstacle. Though reflected point is different from reflector and echo is not reflected at the same point, we assume that echo is reflected at the same point. In many works, ultrasonic transceiver is used to measure distance for simple but accurate characteristics. In general distance error may be within about $2 \sim 3 \%$ of distance from the reflector at most. However, this error is serious in direction angle. From the above equation, $1 \mathrm{~cm}$ distance error leads to about 4 degree direction angle error and $10 \mathrm{~cm}$ lead to as many as about 40 degrees. Hence we use not one TOF but several TOFs and software median filter, to reduce direction angle error.

\section{Binaural Sound Generation}

Auditory sensor of human is the second sensory system that can obtain much information next to visual sensation. Hence we employ the auditory sensory system as the substitution of visual sensory system.

There are two types of information which can be provided with auditory sensation: One is high level abstraction method using language, and the other is low level abstraction method using beep. The language method has advantages that it is possible to communicate accurate information by abstract expression. However, it is difficult to convey information fast, and iterative information conveyance make the user feel fatigue. In the case of beep method, accurate information communication is difficult compared with language method, but it is possible to transmit a large amount of information in very short time, since the user can get the information with intuition. Also the user can make conversation with other persons even while using the system. Hence the beep method is used in our system.

The beep method uses the fact that human can recognize not only features of sound itself but also the spatial position of the sound source using only auditory sensation by the difference of sound received by left and right ears. This comes from the geometrical arrangement of human ears. Hence we use the the sound source as the analogue of the position of obstacle.

In human audition, horizontal discrimination is mainly due to intensity and phase binaural differences [16] and 
vertical discrimination is due to differences in the timbre caused by reflection on the pinna of the ear [17]. Therefore, in our system, directional discrimination is based on the binaural phase difference and the sound intensity, which is part of the inverse model of audition. The beep sounds of low frequencies are assigned for the position data from the cane type system, and the beep sounds of high frequencies for the glasses type system. This choice corresponds to a natural feature in the localization of sounds by the human hearing system.

Signals produced by the inverse model of audition have to satisfy the human auditory system features [18], [19], [20].

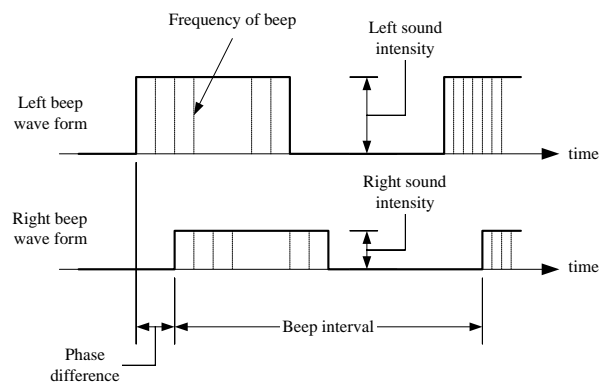

Fig. 6. Beep sound wave forms and parameters

In our system, each item of visual information of obstacle is transformed to the features of sound as Fig. 6.

- The directional information is transformed to binaural phase difference and sound intensity.

- Lower octave musical sound is assigned to the information from the cane type system, and higher octave musical sound is assigned to the information from the glasses type system.

- The distance information is transformed to the frequency of beep. The frequency is inversely proportional to the distance.

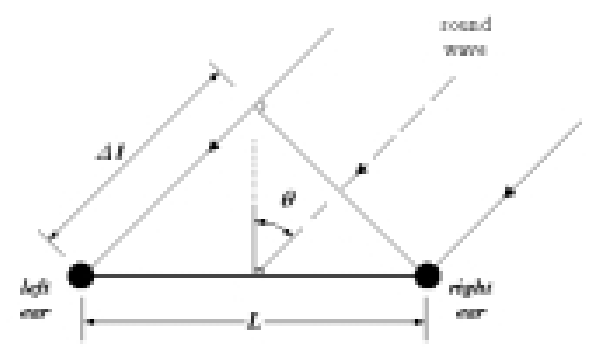

Fig. 7. Modeling of phase difference

We choose the value of binaural phase shifting as the binaural phase difference of our system. The phase difference is determined by the geometrical organization between two ears as Fig. 7. The phase difference $\Delta \Phi$ can be calculated as Eq. 6.

$$
\begin{gathered}
\Delta l=L \cdot \sin \theta \\
\Delta \Phi=\frac{\Delta l}{v_{s}}=\frac{L}{v_{s}} \cdot \sin \theta
\end{gathered}
$$

where $v_{s}$ is the velocity of sound wave in air, and the value is about $344 \mathrm{~m} / \mathrm{s}$.

The maximum phase difference is about $700 \mu \mathrm{sec}$, since the average distance between two ears is about $24 \mathrm{~cm}$.

However, sound intensity of real world varies according to the distance and arrangement between the sound source and the receiver. Moreover, the intensity difference varies in very narrow range. Hence, it is not proper to employ the real intensity difference value. We use the intensity equalization method to augment the intensity effect. The sound intensity value is chosen according to the direction of obstacle, and sifted to the entire intensity range being available as Fig. 8 shows.

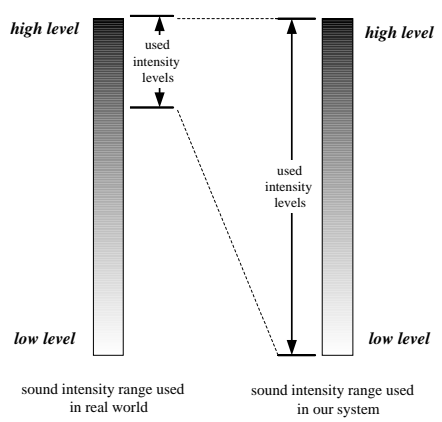

Fig. 8. Sound intensity equalization method

\section{Experiment Results}

In our system, new pulses are transmitted as soon as the echo from the previous pulse is detected. After transmitted, previous pulses are processed before interrupted by the new pulses. Therefore scan interval is $50 \mathrm{~ms}$ which emitted energy can be reached at round-trip distance 10m (including processing time). To evaluate the performance of the developed system, laboratory experiments have been conducted. Four representative objects were chosen to verify the accuracy of the distance measurement. The objects included flat panel of $80 \mathrm{~cm}$ and $60 \mathrm{~cm}$, coal-tar cylinder of $13 \mathrm{~cm}$ diameter, steel pole of $3 \mathrm{~cm}$ diameter, and sweater. As can be seen from Fig. 9, the distance measurement data for the flat panel have shown a low relative error of around $2 \%$. Relative error of cylinder is around $3 \%$ and that of the steel pole can is around 4\%. Maximum relative error of the sweater have shown a high relative error of around $5 \%$. In addition, these experiments show that relative error of distance is related to the size and the quality of the material. Each reflector's detectable distance is about $5 \mathrm{~m}$ for flat panel, $3 \mathrm{~m}$ for the others.

Another experiments was conducted to evaluate the performance of recognizing direction. To reduce the direction angle error, distance and direction angle is computed after 10 scanning and software filtering. Using this method, we can get about 25 degrees to left and right detectable range.

Since Our system is operated by the four $1.2 \mathrm{~V}, 1600 \mathrm{mAh}$, $\mathrm{Ni}-\mathrm{MH}$ batteries and consumes about $210 \mathrm{~mA}$, it can be used for 7.5 hours.

Fig. 10 shows the developed glasses type subsystem. The 


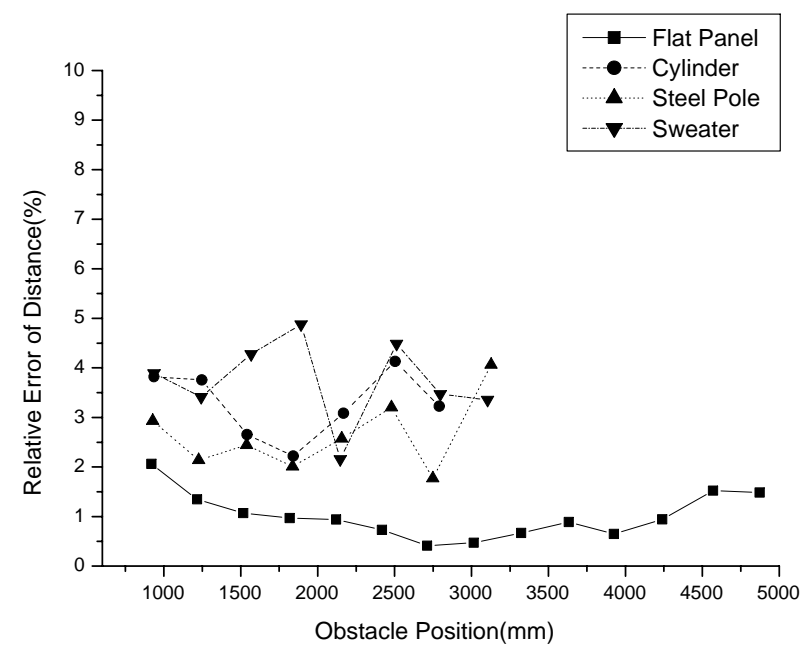

Fig. 9. Measurement error relative to the obstacle distance

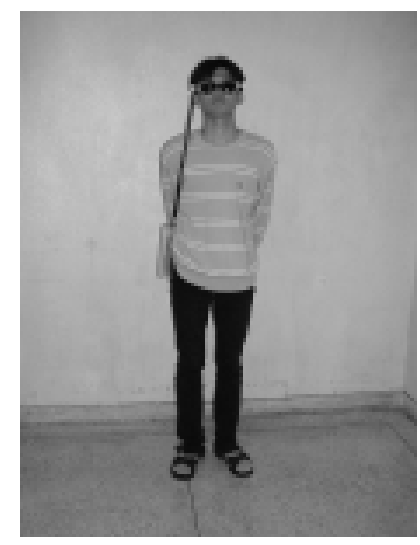

Fig. 10. Glasses type subsystem

user wears the glasses equipped with ultrasonic sensors, and the other equipments such as microproccessor module, interface circuits, and batteries are carried on his belt. This system has reached the clinical testing stage, and system upgrade is in progress based on experimental results.

\section{CONCLUDING REMARKS}

In this paper, we described a signal transformation system from visual information to auditory information using ultrasonic sensors. The broad beam angle ultrasonic sensors enable wide range environment recognition. The main functions of this system are the clear path indication and the environment recognition. Two types of devices are developed to implement these two functions. The visual information acquired by ultrasonic sensors is ultimately transformed to the binaural beep sound. The sound components of frequency, binaural phase difference, and intensity are used to represent the position information of obstacles. We expect that the signal transformation system will reduce the training time to use white stick, and augment the handicapped people's welfare.
If a visually impaired person want to go to a city-hall, he can walk through a road or corridor using an ETA system in local area. However, it is difficult to know where he is globally. Hence, the global positioning method will be studied in our next research. The global position of user is obtained using the Global Positioning System (GPS), the current position and the guidance to the destination will be informed to the user by voice. Also, wall following function will be added so that the blind can walk straight along the corridor in indoor environment.

\section{REFERENCES}

[1] C. J. Robinson, "Through the looking glass: Emerging horizontal in rehabilitation engineering," in Biomedical Engineering, Proceedings of a Special Symposium on Maturing Technologies and Emerging Horizons, 1988, pp. 20-25.

[2] L. Kay, "An ultrasonic sensing probe as a mobility aid for the blind," Ultrasonic, vol. 2, pp. 53, 1964.

[3] J. Barth adn E. Foulhe, "Preview: A neglected variable in orientation and mobility," Journal of Visual Impairment and Blindness, vol. 73, no. 2, pp. 41-48, Feb. 1979.

[4] A. Dodds, D. Clark-Carter, and C. Howarth, "The sonic PathFinder: an evaluation," Journal of Visual Impairment and Blindness, vol. 78, no. 5, pp. 206-207, May 1984.

[5] A. Heyes, "A polaroid ultrasonic travel aid for the blind," Journal of Visual Impairment and Blindness, vol. 76, pp. 199-201, May 1982.

[6] J. D. Armstrong, "Summary report of the research program on electronic mobility aids," Tech. Rep., Dep. Psychology, Univ. Nottingham, Nottingham, England, 1973.

[7] P. W. Nye, "A preliminary evaluation of the bionic instrumentsveterans administration C-4 laser cane," Tech. Rep., National Academy Sci., 1973.

[8] J. Borenstein and I. Ulrich, "The GuideCane - a computerized travel aid for the active guidance of blind pedestrians," in Proceedings of the IEEE International Conference on Robotics and Automation, 1997, vol. 2, pp. 1283-1288.

[9] T. Ifukube, T. Sasaki, and C. Peng, "A blind mobility aid modeled after echolocation of bats," IEEE Transactions on Biomedical Engineering, vol. 38, no. 5, pp. 461-465, May 1991.

[10] S. Shoval, J. Borenstein, and Y. Koren, "Mobile robot obstacle avoidance in a computerized aid for the blind," in Proceedings of the IEEE International Conference on Robotics and Automation, May 1994.

[11] L. Kay, "The design and evaluation of a sensory aid to enhance spatial perception of the blind," Tech. Rep., Dep. Elect. Eng. Univ. Canterbury, New Zealand, Rep. 21, 1973.

[12] L. Kay, "A sonar aid to enhance spatial perception of the blind: Engineering, design and evaluation," Radio and Electronic Engineer, vol. 44, no. 11, pp. 605-627, 1974.

[13] Lawson, "Monotonic compensation filters optimize word recognition for observers with age-related maculopathies," in Optical Society of America Technical Digest Series 3, 1988, pp. 30-33.

[14] Loshin, "Image remapping as a potential aid for patients with peripheral field defects," in Proceedings of the Annual Meeting of the America Academy of Optometry, 1987, p. 18.

[15] J. A. Brabyn, "Design considerations in sensory aids for the visually impaired," in Proceedings of IEEE Conference on Medical Instrument Design, October 1991, pp. 188-199.

[16] W. L. Gulick, G. A. Gescheider, and R. D. Frisina, Hearing Phychoacoustics, Oxford University Press, New York, 1989.

[17] S. K. Roffler and R. A. Butler, "Factors that influence the localization of sound in the vertical plane," Journal of Acoust. Soc. Amer., vol. 43, pp. 1257-1259, 1968.

[18] C. Capelle, C. Trullemans, P. Arno, and C. Veraat, "A real-time experimental prototype for enhancement of vision rehabilitation using auditory substitution," IEEE Trans. on Biomedical Engineering, vol. 45, no. 10, pp. 1279-1293, 1998.

[19] W. F. Ganong, Review of Medical Physiology, Prentice-Hall, Appleton \& Lange, Eds. London, U.K., 15 edition, 1991.

[20] E. Zwicker, "Scaling" in Handbook of Sensory Physiology, vol. V/2 Auditory System, Physiology (CNS), Behavioral Studies, Psychoacoustics, W.D. Keidel and W.D Neff, Eds. Berlin, Germany: Springer-Verlog, 1975. 